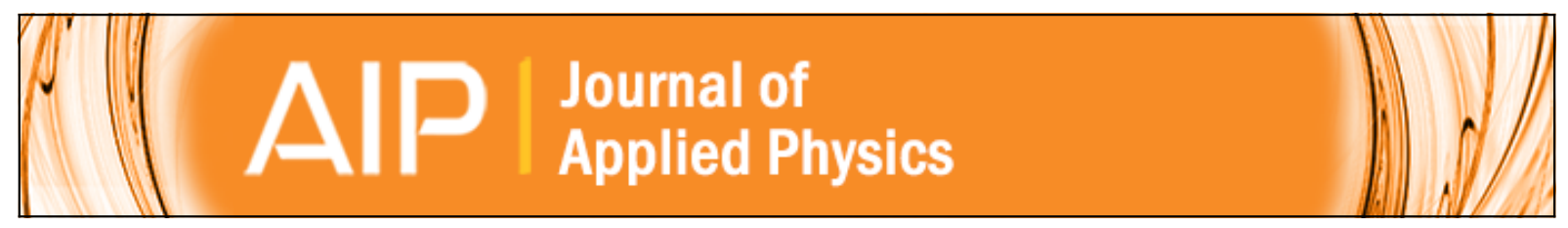

\title{
Many-body effects in wide parabolic AIGaAs quantum wells
}

A. Tabata, M. R. Martins, J. B. B. Oliveira, T. E. Lamas, C. A. Duarte, E. C. F. da Silva, and G. M. Gusev

Citation: Journal of Applied Physics 102, 093715 (2007); doi: 10.1063/1.2809418

View online: http://dx.doi.org/10.1063/1.2809418

View Table of Contents: http://scitation.aip.org/content/aip/journal/jap/102/9?ver=pdfcov

Published by the AIP Publishing

\section{AlP Re-register for Table of Content Alerts}

\section{Create a profile. \\ Sign up today!}




\title{
Many-body effects in wide parabolic AIGaAs quantum wells
}

\author{
A. Tabata, M. R. Martins, and J. B. B. Oliveira \\ Universidade Estadual Paulista, Caixa Postal 473, Bauru, 17033-360 Sao Paulo, Brazil \\ T. E. Lamas, C. A. Duarte, E. C. F. da Silva, ${ }^{\text {a) }}$ and G. M. Gusev \\ Laboratório de Novos Materiais Semicondutores, Instituto de Física da Universidade de São Paulo, \\ CP 66318, 05315-970 São Paulo, Brazil
}

(Received 25 June 2007; accepted 16 September 2007; published online 14 November 2007)

\begin{abstract}
Photoluminescence measurements at different temperatures have been performed to investigate the optical response of a two-dimensional electron gas in $n$-type wide parabolic quantum wells. A series of samples with different well widths in the range of 1000-3000 $\AA$ was analyzed. Many-body effects, usually observed in the recombination process of a two-dimensional electron gas, appear as a strong enhancement in the photoluminescence spectra at the Fermi level at low temperature only in the thinnest parabolic quantum wells. The suppression of the many-body effect in the thicker quantum wells was attributed to the decrease of the overlap between the wavefunctions of the photocreated holes and the two-dimensional electrons belonging to the highest occupied electron subband. () 2007 American Institute of Physics. [DOI: 10.1063/1.2809418]
\end{abstract}

\section{INTRODUCTION}

The realization of systems that can provide a confined carrier gas with very high mobility represents an important step in the semiconductor technology. One key ingredient for the fabrication of such structures is the modulation doping technique, where dopants and the confined carrier gas are spatially separated. From the point of view of transport properties, parabolic quantum wells (PQWs) which are the object of this paper have properties comparable to many other systems designed to the development of high performance devices. ${ }^{1-3}$ However, $\mathrm{Al}_{x} \mathrm{Ga}_{1-x} \mathrm{As} / \mathrm{GaAs} \mathrm{PQWs}$ represent promising systems to the development of spintronics devices by the effective control and manipulation of the spin of the electrons since the Landé $g$ factor varies spatially as a function of the $\mathrm{Al}$ content along the growth direction $z .{ }^{4,5}$ Moreover, modulation of the $g$ factor has been recently demonstrated by the positioning of the wavefunction in lowdimensional devices using front and back gates. ${ }^{6}$ As a consequence, the spin properties (for instance, the spin lifetime) will be also influenced, which allows to fabricate in principle a logic gate for quantum computing.

From the fundamental point of view, PQWs represent interesting systems to investigate collective phenomena such as charge-density waves and spin-density waves at very low temperature and intense applied magnetic fields. ${ }^{7,8}$ Among the collective phenomena, many-body effects of a twodimensional (2D) degenerate electron or hole gas confined in a large variety of semiconductor structures have attracted considerable attention in the literature. ${ }^{9-19}$ Such many-body effects resulting from the rearrangement in the electron and hole systems in order to screen the Coulombic interaction between free carriers cause two major correlation effects, i.e., the band-gap renormalization ${ }^{9-11}$ and the Fermi-edge singularity (FES). ${ }^{11-13,16}$ The FES leads to a strong enhancement of the oscillator strength for the transitions involving



states at the Fermi energy, giving rise to a sharp excitonlike emission in the optical spectra. The FES was predicted theoretically ${ }^{10,12}$ and experimentally demonstrated ${ }^{13-19}$ for an expressive number of semiconductor heterostructures. Two dominant mechanisms have been considered in the literature to be responsible for the FES in III-V quantum wells. ${ }^{13-17}$ One is the effect of the minority carrier localization due to alloy fluctuation and interface roughness, ${ }^{13-15}$ and the other is the scattering between the electronic states near the Fermiedge energy and the next unoccupied subband. ${ }^{12,16,17}$ Both mechanisms explain the contribution of the $k=0$ transitions to states at the Fermi energy $E_{F}$.

In the present work, we report on the optical response of a two-dimensional electron gas (2DEG) in a series of $n$-type wide parabolic quantum wells (WPQWs). We have observed strong luminescence associated to the confined electron gas in samples with different well widths $(1000,1500,2000$, and $3000 \AA$ ) and a pronounced enhancement in the high-energy cutoff in the spectrum of the thinnest samples. The analysis of our data based on self-consistent calculations indicates that the close position of the Fermi level to the empty electron subband is the mechanism responsible for the development of the FES observed in the thinnest samples. Our findings also indicate that a reduction of the overlap between the wavefunction of electrons close to the Fermi energy and the hole wavefunction is responsible for the disappearance of the FES in the thickest samples.

\section{SAMPLE AND EXPERIMENTAL DETAILS}

All the samples here investigated were grown in a Gen II molecular beam epitaxy (MBE) system on top of epiready semi-insulating GaAs(001) substrates. We used a substrate temperature of $580{ }^{\circ} \mathrm{C}$ and an $\mathrm{As}_{4} /(\mathrm{Ga}+\mathrm{Al})$ flux ratio as low as possible $(\sim 1.2)$ but still consistent with a good epilayer morphology. The structure of the PQWs is shown in Fig. 1. First, a $1-\mu$ m-thick GaAs buffer was grown with a 20 $\times(\mathrm{AlAs})_{5}(\mathrm{GaAs})_{10}$ superlattice after the growth of the first 


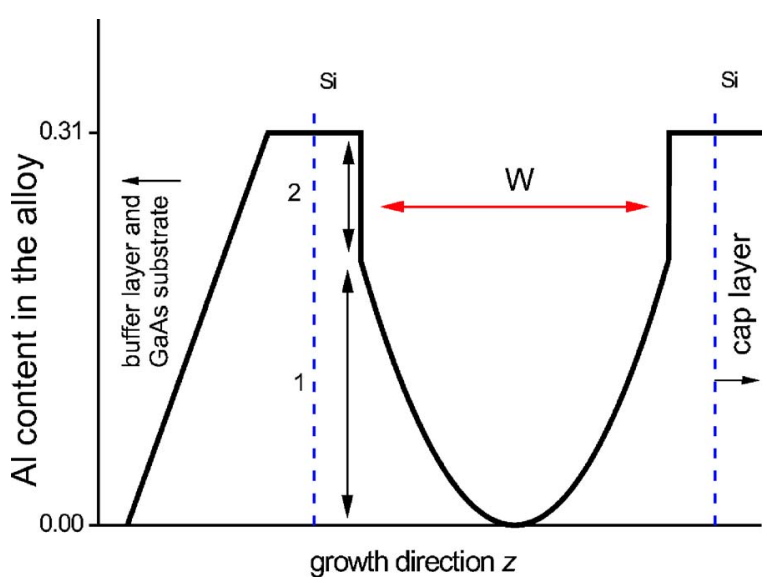

FIG. 1. (Color online) Aluminum concentration profile the samples along the growth direction. $W$ is the well width, $\Delta_{1}$ is the depth of the parabolic profile, and $\Delta_{2}$ was used to increase the barrier height and is equal to $88.7 \mathrm{meV}$ for the 1000 -Å-thick PQW and to $32.7 \mathrm{meV}$ for the other samples. The sum $\Delta_{1}+\Delta_{2}$ is constant and equal to $236.16 \mathrm{meV}$.

$2000 \AA$ in order to improve the crystal purity and quality. Then a 500- $\AA$-thick AlGaAs layer was grown with the $\mathrm{Al}$ content varying linearly from $0 \%$ to $31 \%$ using the digitalalloy technique, after which it was grown a $\mathrm{Al}_{0.31} \mathrm{Ga}_{0.69} \mathrm{As}$ layer $1000 \AA$ thick. Then, $\mathrm{Al}_{x} \mathrm{Ga}_{1-x} \mathrm{As}$ parabolic quantum wells were grown with the $\mathrm{Al}$ content varying from $x=0$ in the center of the parabola to $x=0.20(x=0.27)$ at the edges of the parabola for a well width below $1500 \AA$ (for a well width equal or larger than $1500 \AA$ ). The parabolic wells were surrounded by $\mathrm{Al}_{0.31} \mathrm{Ga}_{0.69}$ As barriers containing two Si spikes symmetrically located at $200 \AA(150 \AA)$ from the borders of the well for the sample(s) with $1000 \AA(\geqslant 1500 \AA)$ well width(s). The nominal Si concentration was $1 \times 10^{12} \mathrm{~cm}^{-2}$ for the sample with the 3000 - $\AA$-wide parabolic well and 5 $\times 10^{11} \mathrm{~cm}^{-2}$ for all the remaining ones. Inside the well, the parabolic potential profile was achieved by the digital-alloy technique using a $20-\AA$-period superlattice in which the respective thickness of GaAs and AlAs were varied accordingly. Then it was grown a cap layer consisting of a $400 \AA$ $\mathrm{Al}_{0.31} \mathrm{Ga}_{0.69} \mathrm{As}$, a third $\mathrm{Si}$ delta-doped layer $(2.8$ $\times 10^{12} \mathrm{~cm}^{-2}$ ) to saturate the surface dangling bonds, followed by a $200 \AA \mathrm{Al}_{0.31} \mathrm{Ga}_{0.69}$ As layer and a final $100 \AA$ GaAs layer. Reference samples with similar structures were also grown without the Si spikes in the barriers of the wells. The low temperature $(1.5 \mathrm{~K})$ mobility of the electron gas in the doped samples was about $80 \times 10^{3} \mathrm{~cm}^{2} / \mathrm{V} \mathrm{s}$. The Hall electron concentration (measured under illumination condition) was 5.8, 5.6, 4.5, and $3.5 \times 10^{11} \mathrm{~cm}^{-2}$ for the samples with increasing well width, respectively. ${ }^{2}$ The reason why the concentration of the 2DEG varies from sample to sample is mainly due to the different widths of the quantum wells. By increasing the width of the quantum well, the energy separation of the energy levels decreases and the number of occupied levels increases. In this way, the amount of carriers that will be transferred from the delta-doped layers to the parabolic wells changes because of the principle which imposes that the Fermi level must be constant in the entire heterostructure. In other words, the amount of charge transferred from the Si delta-doped layers is a self-consistent problem: it

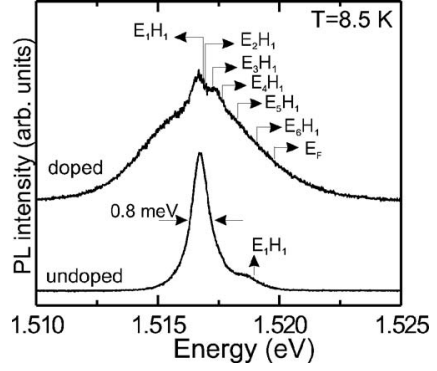

FIG. 2. Low temperature PL spectra of the undoped (reference) and doped WPQWs with geometric well width of $3000 \AA$. Indicated by arrows are shown the calculated values of the recombination energies of electrons in the $i$ th subband with photogenerated holes in the first hole subband, i.e., the recombination energies $E_{i} H_{1}$.

depends of the electronic structure of quantum well which depends on the amount of charge transferred among the different regions of the sample.

Photoluminescence (PL) measurements were performed in a closed circuit optical cryostat operating with helium from 8.5 to $30 \mathrm{~K}$. The samples were excited with the $5145 \AA$ line of an argon laser with a maximum excitation power of $35 \mathrm{~mW} / \mathrm{cm}^{2}$. The luminescence signal was analyzed by a monochromator and detected by a cooled charge coupled device $(\mathrm{CCD})$.

In order to analyze our data, calculations of the band bending, the subband energy, subband occupation, and envelope wavefunctions for the thinnest and thicker quantum wells were performed. Quantized energy levels were calculated by simultaneously and self-consistently solving Schrödinger and Poisson equations within the context of the effective-mass formalism. The exchange and correlation effects were included in the self-consistent calculations by means of a local density approximation. ${ }^{20}$ The input to the self-consistent calculations comprises parameters related to the AlGaAs alloy (effective mass, dielectric constant, and band gap), the width of the spacer, and the electron concentration (electron sheet density $n_{s}$ ) confined in the wells.

\section{RESULTS AND DISCUSSION}

Figure 2 shows the low temperature $(8.5 \mathrm{~K}) \mathrm{PL}$ spectra for an undoped (reference) and a doped sample with $3000 \AA$ well width. We can see that the PL spectrum of the undoped sample consists of one main peak with a small shoulder on the high-energy side. The structure at $1.516 \mathrm{eV}$ with a full width at half maximum of $0.8 \mathrm{meV}$ is an excitonic emission of the GaAs material. The figure also shows (indicated by an arrow) the theoretical position of the fundamental transition involving photogenerated electrons and holes in the first electron and hole subbands (the $E_{1} H_{1}$ energy), which agrees with the high-energy side shoulder observed in the PL spectrum of the undoped sample. A huge difference can be noticed in the PL spectrum of the doped sample, where a relatively broad $(\sim 4.5 \mathrm{meV})$ band is observed. The figure shows the expected value of the recombination energy $E_{1} H_{1}$ as well as the higher transition energies $E_{i} H_{1}$, with $i=2,3,4,5$, and the position of the Fermi energy $E_{F}$. Only the transitions involving the heavy holes were considered because the experiments were done in low temperature using very low laser 


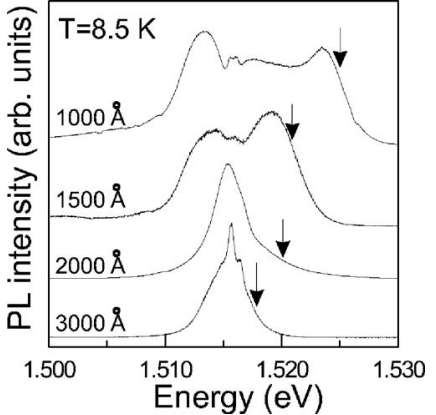

FIG. 3. Low temperature PL spectra of doped PQWs with different well thicknesses. The arrows indicate the theoretical position of the Fermi level.

excitation power. Another reason why only the first hole subband was considered in our analysis is because, due to valence band mixing effects and the small spacing in energy between confined levels, a very efficient thermalization process of photogenerated holes is expected. Based on these arguments and also on the self-consistent results, we ascribe the high-energy side of the spectrum to transitions involving the different subbands populated by the 2DEG and holes in the first hole subband. As we shall see latter (in Fig. 4), the behavior of the low-energy side broadening as a function of temperature observed in the spectrum of the $3000 \AA$ doped sample suggests an extrinsic origin.

Figure 3 shows the low temperature PL spectra for the doped parabolic quantum wells with different well thicknesses from 1000 to $3000 \AA$. The arrows indicate the theoretical values of $E_{F}$ for each sample. In all samples, the GaAs emission is present at $1.516 \mathrm{eV}$. For some samples, an emission below the GaAs excitonic emission is observed at $1.513 \mathrm{eV}$ (see in Fig. 3 the broadband in the PL spectra of the sample with $1000 \AA$ well width). Measurements of PL as a function of temperature and photoexcitation intensity show that this emission has a typical extrinsic behavior: it disappears at high temperatures and saturates with increasing excitation intensity. The energy of this peak suggests that it can be interpreted as arising from the recombination of electrons trapped by residual donors (silicon atoms) inside the parabolic quantum well and confined photogenerated holes. Although there exist other recombination processes which could be assigned to this peak (see, for instance, the $\mathrm{H}$ band described in Ref. 21), our interpretation is based on the fact that, during the growth process, $\mathrm{Si}$ atoms in the atmosphere of the growth chamber can be incorporated as a residual impurity inside the PQWs as already observed for the growth of samples with similar structure to the ones analyzed here. ${ }^{22}$ Moreover, it is important to notice that the PL spectra of the undoped samples (see Fig. 2) does not show any emission peak or any low energy broadening around the energy of $1.513 \mathrm{eV}$ (with exception of the intrinsic emission of the GaAs), which reinforces the hypothesis that this peak has extrinsic origin.

Before continuing our analysis, we would like to discuss the shape of the spectra of the thinnest sample (the PQW with $1000 \AA$ ). For this purpose, we performed PL measurements as a function of temperature (from 8.5 to $30 \mathrm{~K}$ ) and the obtained spectra are shown in Fig. 4. At low temperature,

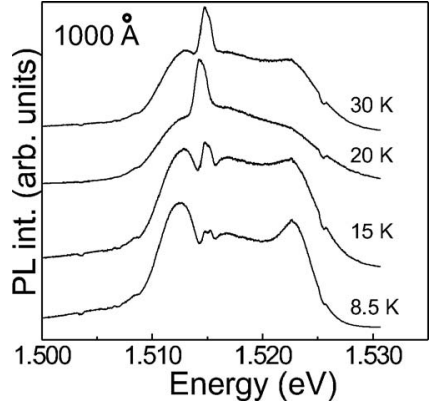

FIG. 4. PL spectra as a function of temperature of the WPQW with $1000 \AA$ well width.

the dominant peak at $1.513 \mathrm{eV}$ corresponds to the recombination of electrons trapped by residual donors and photogenerated free holes. As mentioned before, this emission saturates with the increase of the excitation power (not shown here) in agreement with extrinsic impurity behavior. The remaining broad luminescence is interpreted as follows: according to our self-consistent calculations, three electron subbands are occupied for this sample. So, we ascribe the broad emission band to the recombination processes of electrons from the 2DEG with holes at the first hole subband. Moreover, this broad luminescence at low temperature condition is only possible if $k$ conservation does not restrict the participation of all the electrons of the Fermi gas in the recombination process. This condition is ensured because the holes are strongly localized in real space and have sufficient spread of $k$ vector to enable electron up to $k=k_{F}$ to recombine without significant restriction due to $k$ conservation. In the present case the holes in the AlGaAs parabolic wells are sufficiently localized, probably due to alloy fluctuations of the digital alloy and/or interface roughness.

We also observe in Fig. 4 that, at low temperature, the broad band has a sharp high-side cutoff and an enhanced recombination near the Fermi edge, which is a characteristic feature of the well reported FES due to many-body effects. ${ }^{13}$ The enhancement effect associated to the FES can be strongly reduced with increasing temperature, as depicted in Fig. 4. This thermal quenching behavior is characteristic for the FES due to the thermal broadening of the Fermi edge and well documented for III-V modulation doped quantum wells. $^{12-17}$

The FES is not observed on the thickest samples, as can be seen in Fig. 3. Theory predicts ${ }^{12}$ that the FES can be largely suppressed if the overlap between the wavefunction of the electron of the Fermi sea and the photogenerated hole is weak. To examine whether this is the case for the wider PQWs, we performed theoretical calculations in order to obtain the electronic structure of the PQWs. Figure 5 shows these theoretical results (potential profile, the electron distribution function, and the envelope wavefunctions) for the thinnest (1000 $\AA$ ) and the thickest (3000 $)$ ) samples. We can see that, for the 1000 - $\AA$-thick well, the electron distribution is almost constant in the central part of the well and the bottom of the conduction band is almost flat as a square well [Fig. 5(a)]. In this case, the electrons in the conduction band screen very effectively the parabolic potential forming a 


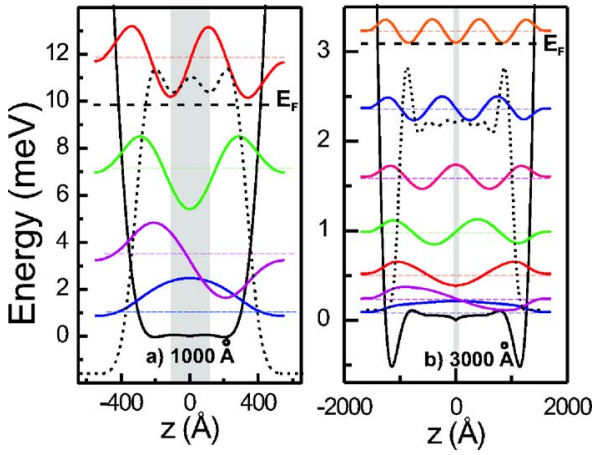

FIG. 5. (Color online) Relevant portion of the conduction band profile with subband structure and envelope wavefunctions for (a) the thinnest (1000) and (b) for the thicker (3000 $)$ ) PQWs. Only the region very close to the bottom of the conduction band is shown in order to show the normalized envelope wavefunctions of electrons. The horizontal dashed line indicates the Fermi level position and the dashed curve is the electron distribution function. The vertical shady areas indicate the half-width $(\delta z)$ of the hole wavefunctions.

2DEG. However, for the 3000- $\AA$-thick well, the selfconsistent potential shows dips (with an almost triangular shape) at the interfaces of the well [see Fig. 5(b)]. As the Fermi level is located just few meV above the flat part of the potential well, part of the electrons drift to the interfaces. This is inferred from the self-consistent calculations which show maxima on the electron distribution profile close to the interfaces, as can be seen by the dashed line in Fig. 5(b). Thus, for this sample there is a considerable part of electrons localized at the interfaces. We also verified that the envelope wavefunction of the holes in the first subband has an almost Gaussian shape with a half-width $\delta z=200 \AA$ for the 3000- $\AA$-thick well and $\delta z=160 \AA$ for the 1000 - $\AA$-thick well and with a maximum located in the center of the well.

The self-consistent solutions for the lowest subbands (three occupied and one empty) for the 1000- $\AA$-thick well with $n_{\mathrm{s}}=5.8 \times 10^{11} \mathrm{~cm}^{-2}$ are $E_{1}=0.854 \mathrm{meV}, E_{2}=3.23 \mathrm{meV}$, $E_{3}=6.96 \mathrm{meV}, E_{4}=10.846 \mathrm{meV}$, and $E_{F}=9.85 \mathrm{meV}$. We adopted the origin of the energy scale at the bottom of the potential well at $z=0$. The calculated occupancies for the three occupied subbands are $n_{1}=2.71 \times 10^{11} \mathrm{~cm}^{-2}, n_{2}=2.06$ $\times 10^{11} \mathrm{~cm}^{-2}$, and $n_{3}=1.03 \times 10^{11} \mathrm{~cm}^{-2}$. For the 3000 - $\AA$-thick well with $n_{\mathrm{s}}=3.5 \times 10^{11} \mathrm{~cm}^{-2}$, we obtained $E_{1}=0.09 \mathrm{meV}, \quad E_{2}=0.24 \mathrm{meV}, \quad E_{3}=0.52 \mathrm{meV}, \quad E_{4}$ $=0.98 \mathrm{meV}, \quad E_{5}=1,60 \mathrm{meV}, \quad E_{6}=2,37 \mathrm{meV}, \quad n_{1}=8.25$ $\times 10^{10} \mathrm{~cm}^{-2}, n_{2}=7.83 \times 10^{10} \mathrm{~cm}^{-2}, n_{3}=7.07 \times 10^{10} \mathrm{~cm}^{-2}, n_{4}$ $=5.79 \times 10^{10} \mathrm{~cm}^{-2}, \quad n_{5}=4.08 \times 10^{10} \mathrm{~cm}^{-2}, \quad$ and $n_{6}=1.99$ $\times 10^{11} \mathrm{~cm}^{-2}$ for the six occupied subbands. We also found $E_{F}=3.09 \mathrm{meV}$, and for the first empty subband $E_{7}$ $=3.23 \mathrm{meV}$. We observe that the Fermi level in each structure lies very close to the next unpopulated electron subband.

In order to understand why the spatial separation of electrons and holes leads to the suppression of the FES, it is important to look in more details the electron wavefunctions of the PQWs. Two dominant mechanisms have been considered in the literature to be responsible for the FES in modulation-doped III-V quantum wells: (i) localization of the minority carriers or (ii) a small energy separation between the Fermi level and the next unoccupied electron subband providing a resonant condition for efficient scattering of the 2DEG. The former condition is realized for all the samples here analyzed since the holes can be localized either by alloy fluctuation or heterointerface roughness of the digital alloy. The later condition is also fulfilled for all the samples analyzed in this work since the quantum wells are so wide that the Fermi level $E_{F}$ is always close to the next empty electron subband within a few $\mathrm{meV}$, where the scattering of electrons at the Fermi level by holes is enhanced due to interaction with nearly resonant excitons related to the empty subband. However, the FES is not observed for the thicker parabolic wells where the reduction in the energy separation between $E_{F}$ and the next unoccupied subband is more pronounced. Thus, we can conclude that even the simultaneous fulfillment of the two aforementioned conditions is insufficient for the observation of the FES in the PL spectra of the thickest samples. The reason why the FES does not develop in the thickest PQWs is because the expected enhanced interaction between the 2DEG and photogenerated holes is not only ensured by the close distance between the Fermi level and the empty upper subband. The enhancement is large when the overlap between the wavefunctions of the photocreated holes and the $2 \mathrm{D}$ electrons belonging to the highest occupied electron subband is strong. That is just the opposite condition we find in the 3000 - $\AA$-thick PQW, where the wavefunction of the highest occupied subband (the sixth subband) has a node in the center of the well. So, we attribute the reduction of the overlap between the wavefunction of electrons close to the Fermi energy and the hole wavefunctions as being responsible for the disappearance of the FES in the thickest samples.

\section{CONCLUSION}

We have investigated the influence of the many-body effects on the PL properties of a series of wide parabolic quantum wells with different well widths and electron concentrations. The PL spectra showed broad emission bands related to the recombination process of the Fermi gas with photogenerated holes. An increase of the luminescence intensity near the quasi-Fermi level was only observed in the samples with the thinnest parabolic wells. Our results indicate that the mechanism responsible for the observed FES is the efficient scattering between the states at the Fermi energy and the adjacent unoccupied subband since the requirement of hole localization is realized in all the structures here analyzed. The FES was not observed in the thickest samples. We attributed the disappearance of the FES with increasing well width to a reduction of the overlap between the wavefunctions of the photocreated holes and the two-dimensional electrons belonging to the highest occupied electron subband.

\section{ACKNOWLEDGMENTS}

The authors would like to thank the Brazilian Agencies CNPq and FAPESP for partial financial support.

${ }^{1}$ S. A. Studenikin, A. V. Chaplik, I. A. Panaev, G. Salis, K. Ensslin, K. Maranowski, and A. C. Gossard, Semicond. Sci. Technol. 14, 604 (1999). ${ }^{2}$ G. M. Gusev, A. A. Quivy, T. E. Lamas, J. R. Leite, A. K. Bakarov, A. I. Toropov, O. Estibals, and J. C. Portal, Phys. Rev. B 65, 205316 (2002). 
${ }^{3}$ T. E. Lamas, A. A. Quivy, C. S. Sergio, G. M. Gusev, and J. C. Portal, J. Appl. Phys. 97, 076107 (2005).

${ }^{4}$ C. Weisbuch and C. Hermann, Phys. Rev. B 15, 816 (1997).

${ }^{5}$ P. Pfeffer and W. Zawadzki, Phys. Rev. B 72, 035325 (2005).

${ }^{6}$ G. Salis, Y. Kato, K. Ensslin, D. C. Driscoll, A. C. Gossard, and D. D. Awschalom, Nature (London) 414, 619 (2001).

${ }^{7}$ V. Celli and N. D. Mermin, Phys. Rev. 140, A839 (1965).

${ }^{8}$ H. Fukuyama, Solid State Commun. 26, 783 (1978).

${ }^{9}$ C. Delalande, G. Bastard, J. Orgonasi, J. A. Brum, H. W. Liu, M. Voos, G. Weimann, and W. Schlapp, Phys. Rev. Lett. 59, 2690 (1987).

${ }^{10}$ S. Schmitt-Rink, C. Ell, and H. Haug, Phys. Rev. B 33, 1183 (1986)

${ }^{11}$ G. Livescu, D. A. B. Miller, D. S. Chemla, M. Ramaswamy, T. Y. Chang, N. Sauer, A. C. Gossard, and J. H. English, IEEE J. Quantum Electron. 24, 1677 (1988).

${ }^{12}$ P. Hawrylak, Phys. Rev. B 44, 3821 (1991); 44, 6262 (1991).

${ }^{13}$ M. S. Skolnick, J. M. Rorison, K. J. Nash, D. J. Mowbray, P. R. Tapster, S. J. Bass, and A. D. Pitt, Phys. Rev. Lett. 58, 2130 (1987).
${ }^{14}$ Y. F. Chen, L. Y. Lin, J. L. Shen, and D. W. Liu, Phys. Rev. B 46, 12433 (1992).

${ }^{15}$ Y.-H. Zhang, D.-S. Jiang, and K. Ploog, Appl. Phys. Lett. 60, 2264 (1992).

${ }^{16}$ W. Chen, M. Fritze, A. Nurmikko, D. Ackley, C. Colvard, and H. Lee, Phys. Rev. Lett. 64, 2434 (1990).

${ }^{17}$ M. S. Skolnick, D. W. Whittaker, P. E. Simmonds, T. A. Fisher, M. K. Saker, J. M. Rorison, R. S. Smith, P. B. Kirby, and C. R. H. White, Phys. Rev. B 43, 7354 (1991).

${ }^{18}$ I. A. Buyanova, T. Lundstrom, A. V. Buyanov, and W. M. Chen, Phys. Rev. B 55, 7052 (1997).

${ }^{19}$ M. Fritze, W. Chen, A. V. Nurmikko, J. Jo, M. Santos, and M. Shayegan, Phys. Rev. B 45, 8408 (1992).

${ }^{20}$ L. Hedin and B. I. Lundqvist, J. Phys. C 4, 2064 (1971).

${ }^{21}$ Y. R. Yan, M. A. A. Pudensi, G. A. Vawter, and J. L. Merz, J. Appl. Phys. 58, 397 (1985)

${ }^{22}$ G. Salis, P. Wirth, T. Heinzel, T. Ihn, K. Ensslin, K. Maranowski, and A. C. Gossard, Phys. Rev. B 59, R5304 (1999). 